\title{
STUDIES ON THE POTENTIAL OSCILLATION APPEARING IN THE OLFACTORY EPITHELIUM OF THE TOAD
}

\author{
Sadayuki F. TAKAgi AND Tatsuaki ShibuYA* \\ Department of Physiology, School of Medicine, Gunma University, Maebashi
}

In 1956, OtToson found a rhythmic wave appearing superimposed on the slow potential when the olfactory epithelium of a frog was stimulated with an odorous vapour. It was said that they appeared infrequently, and usually some time after dissection, and that they often did after mechanical manipulation. In the previous papers (TAKAGI \& ShIBUYA, 1960 b, c, d), it was stated that in the toad similar potential oscillations appear very frequently with remarkable magnitude especially in winter and spring, and they are inhibited by cocain, but reappear when the epithelium is washed with Ringer's solution. In this paper, it is intended to clarify the various properties and the mechanism of the potential oscillation in the toad. The origin of the oscillation is considered.

\section{METHODS}

Materials. Toads, Bufo vulgaris japonicus, were used. For comparison, some experiments were performed in the frog. Under ether narcosis, the animals were decapitated and the olfactory epithelium, nerve and bulb were exposed.

Kinds of odours. Amyl acetate, amyl alcohol and butyl alcohol were used as stimulants. The saturated vapours of these odorants were diluted to $1 / 2,1 / 4,1 / 8$ and $1 / 16$ by adding pure air obtained through active carbon.

Stimulating apparatus. The above vapours were drawn into $20 \mathrm{cc}$ syringes. For stimulation, the vapours were pushed out at the rates of $0.5 \mathrm{cc}, 0.4 \mathrm{cc}$ and $0.2 \mathrm{cc} / \mathrm{sec}$ by means of a DC motor and were applied onto the whole surface of the olfactory epithelium through a lucite tube of $0.7 \mathrm{~mm}$ inside diameter. The tip of the tube was always kept at a distance of $1 \mathrm{~cm}$ from the epithelium.

Recording apparatus. Glass pipettes with tip diameter of about $100 \mu$ were filled with Ringer's solution, and were used as recording macro-electrodes. As micro-electrodes, $\mathrm{KCl}$-filled glass pipettes with tip diameters of less than $0.5 \mu$ and resistances of 20 to $50 \mathrm{M} \Omega$ were used. A pencil type electrode deviced by TомітA (1956) was used to record potentials inside a small segregated area of the olfactory eminentia, when the rest of the eminentia was stimulated. The outer glass pipette used to segregate a small area had a diameter of $1.5 \mathrm{~mm}$ and the inner one that of $100 \mu$. The potential oscillations were recorded with a four and a six elements ink-writing recorders. A

Received for publication July 29, 1960

* 高木貞敬, 濰谷達明 
DC amplifier and RC coupled amplifiers with a time constant of $1.5 \mathrm{sec}$ were used.

Sometimes, the action potentials were simultaneously recorded in the olfactory bulb to clarify their relation with the potential oscillation in the olfactory epithelium.

\section{RESULTS}

I. The magnitude, shape and frequency; The potential oscillations showed the magnitudes of several hundred micro-volts to more than $5 \mathrm{mV}$. They appeared small at first, but rapidly increased their magnitudes reaching maximum ones. In the end, they decreased their magnitudes rapidly and disappeared. They showed various shapes (FIG. 1). They were often very regular and spindle-shaped or beat-shaped, showing many loops and nodes. In other cases, they were not regular, showing random shapes and irregular magnitudes. Even in the same preparation, the shapes were not stable but became different at each stimulation.

It was stated that the frequencies of the potential oscillations decreased towards the end during a stimulation (TAKagi \& Shibuya, $1960 \mathrm{~d}$ ). But in recent experiments, it was often found that the frequency increases at first and then decreases. The decrease of the frequency was linear in some oscillations but positively or negatively exponential in other ones. It is interesting that such different ways of increase and decrease were found among the oscillations

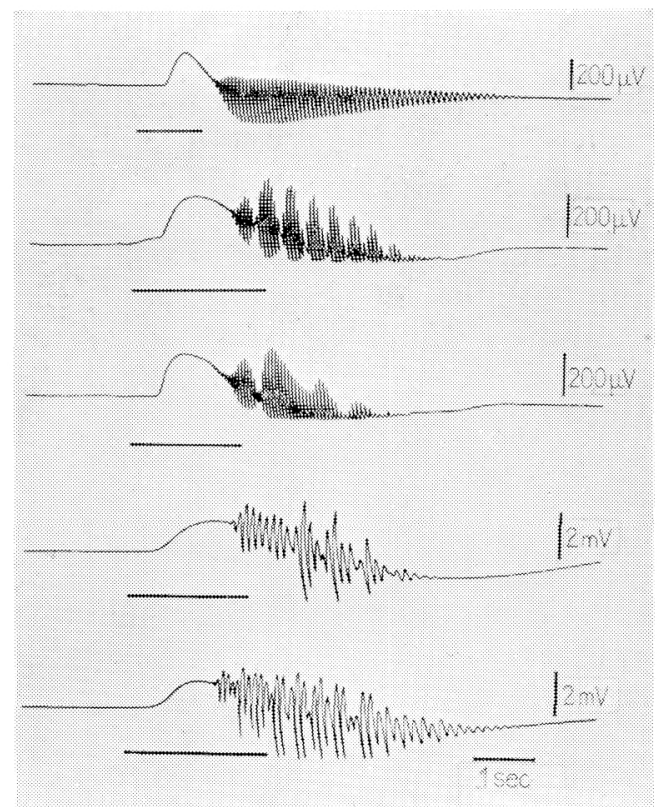

FIG. 1. Various shapes of the potential oscillations. The horizontal line under the slow potential indicates the application of an odorous vapour. 
recorded simultaneously at many points on the same eminentia (FIG. 2). The irregularly shaped oscillations as shown in FIG. 1 may be produced by the interaction of many elementary oscillations which decrease their frequencies in different ways.

II. Recording at various points on the olfactory epithelium; It was shown before (TAKAgI \& SHibuYA, 1960 a) that the slow potentials have different shapes and magnitudes at different points on the epithelium. A similar difference was found with the potential oscillations (FIG. 2). They appeared bigger at the medial-central area and also at the anterior area of the epithelium than at the other area. Moreover, their shapes, durations, and frequencies were different, depending on recording sites. The times of beginning and the modes of changes of the frequencies were also different. From these results, it is conceived that the potential oscillations of various shapes and frequencies are produced by the interaction and summation of some elementary oscillations which occur with different magnitudes, shapes, frequencies and modes of decrease of frequency at different area. The simple, spindle-shaped oscillation shown in FIG. 1 may be one of such elementary oscillations.

III. Intensity of odour; It was reported that the slow potential of the olfactory epithelium increases its magnitude with increasing stimulus intensity (OTTOSON, 1956), (FIG. 3). It was found that the potential oscillation appears when the

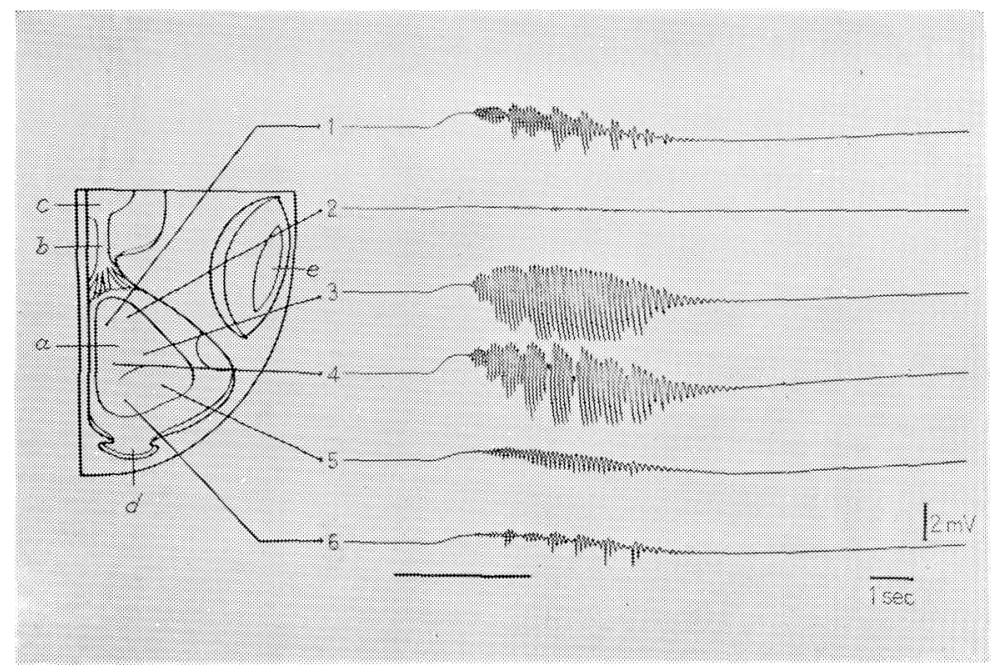

FIG. 2. Simultaneous recording at six points on the olfactory eminentia. In the schematic diagram on the left, $a$ is an olfactory eminentia, $b$ is an olfactory nerve, $c$ is an olfactory bulb, $d$ is a nostril and $e$ is an eyeball. The oscillations appear larger in the medial and central area. The magnitudes, frequencies, shapes, durations and times of appearance are different at each point. A horizontal line in the bottom indicates the application of amyl acetate vapour. 
intensity of an odorous vapour is increased to a certain value and then the oscillation increases its magnitude with increasing intensity (FIG. 3). Even the shapes became different and the frequencies increased in many cases. It is an important finding that the oscillation appears only when the slow potential is enlarged beyond a certain magnitude.

$I V$. Velocity of an odour; Similar phenomena were observed when the velocity of application of an odour was increased. When an odorous vapour of low concentration applied at low speed did not produce any potential oscillation, the same vapour applied at higher speed produced it. In this case also, the slow potential increased its magnitude with increasing velocity and attained a certain magnitude, when the oscillation appeared. It is well known that the intensity of olfactory sensation depends upon the velocity of an odorous air current which flows along the olfactory epithelium (TAKAgI, SHibuya, Higashino \& ARAI, 1960). Therefore, these phenomena are essentially the same as in the above case $I I I$.

$V$. Duration of stimulation; The potential oscillation was prolonged when the duration of stimulation was increased (FIG. 4). However, there was found an upper limit in the potential duration, beyond which the oscillation was never prolonged. The limit varied depending on materials, but under the limit an

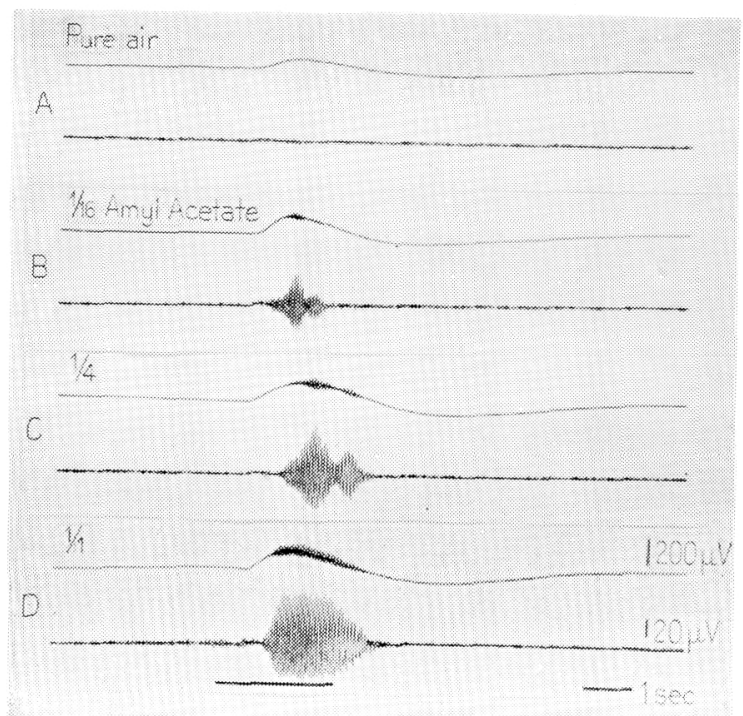

FIG. 3. The intensity of an odour. Pure air in A, 1/16 amyl acetate vapour in B, $1 / 4$ amyl acetate vapour in $C$ and saturated amyl acetate vapour in $\mathrm{D}$ were applied respectively. The oscillation began to appear in $\mathrm{B}$ and increased its amplitude with increasing stimulus intensity in C and D. A horizontal line in the bottom indicates olfactory stimulation. The top and bottom records in each group were obtained respectively by means of amplifiers with a long time constant and low gain and the one with a short time constant and high gain. 


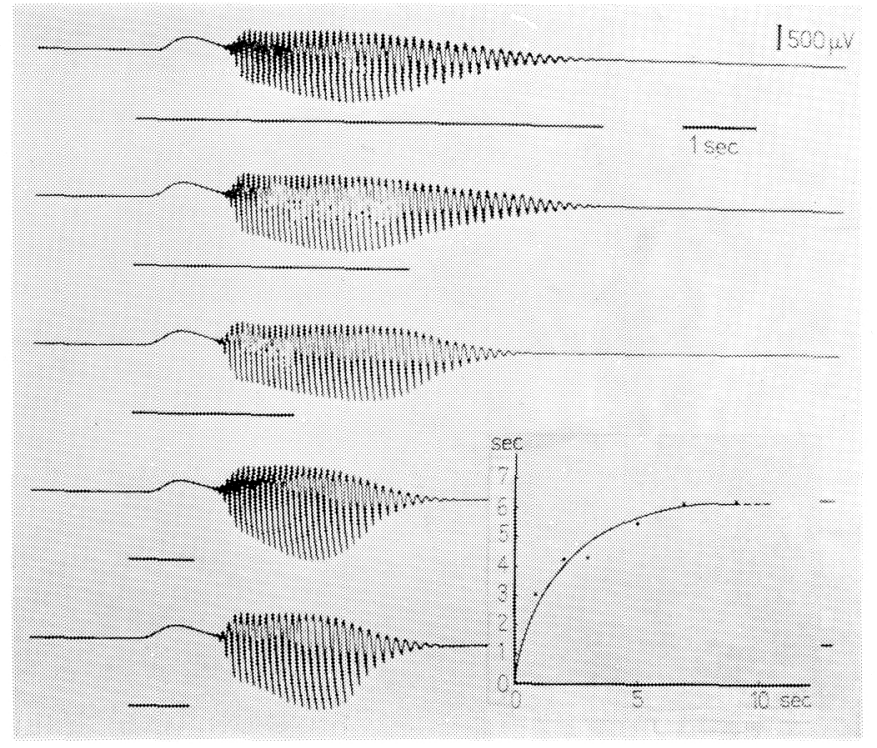

FIG. 4. The duration of an odour. When the duration of stimulation is increased, the duration of the oscillation is increased up to a certain limit. The horizontal line on the left below indicates olfactory stimulation. The inset shows an exponential relation between the two. The ordinate shows the duration of potential oscillation and the abscissa does that of stimulation.

exponential relation was found between the duration of the oscillation and that of stimulation (FIG. 4).

VI. Repetitive stimulation; When repetitive olfactory stimulation was applied, the magnitude and duration of the oscillation gradually decreased, the appearance was often delayed and the shapes became different in many cases (FIG. 5). Moreover, the frequency of the oscillation also decreased during repetitive stimulation. In FIG. 5, the initial frequency of $10 / \mathrm{sec}$ in the first oscillation decreased to $7.7 / \mathrm{sec}$ in the second one which was produced 15 seconds after the first one.

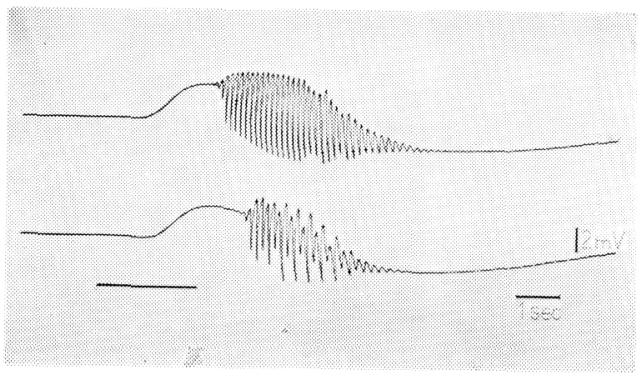

FIG. 5. Repetitive stimulation. Top: control. Bottom: the potential oscillation recorded $15 \mathrm{sec}$ after the top record. Remarkable fatigue phenomena are observed. Detailed explanation in the text. A horizontal line in the bottom indicates olfactory stimulation. 
Accordingly, the terminal frequency also decreased from $5.7 / \mathrm{sec}$ to $5 / \mathrm{sec}$. These phenomena are the more striking, the shorter the interval is made. Coincidentally, the slow potential decreased its magnitude in the second stimulation. Thus, a parallel relation was found between the magnitude of the slow potential and the frequency and magnitude of the potential oscillation.

VII. Kind of odours; It was found that the magnitudes, shapes, durations and sometimes frequencies of the oscillations are different, depending on the kinds of odours. But no definite relation was found between these properties and the kinds of odours.

When the potential oscillation and the slow potential disappeared after repetitive application of an odour, the effects of other kind of odours were

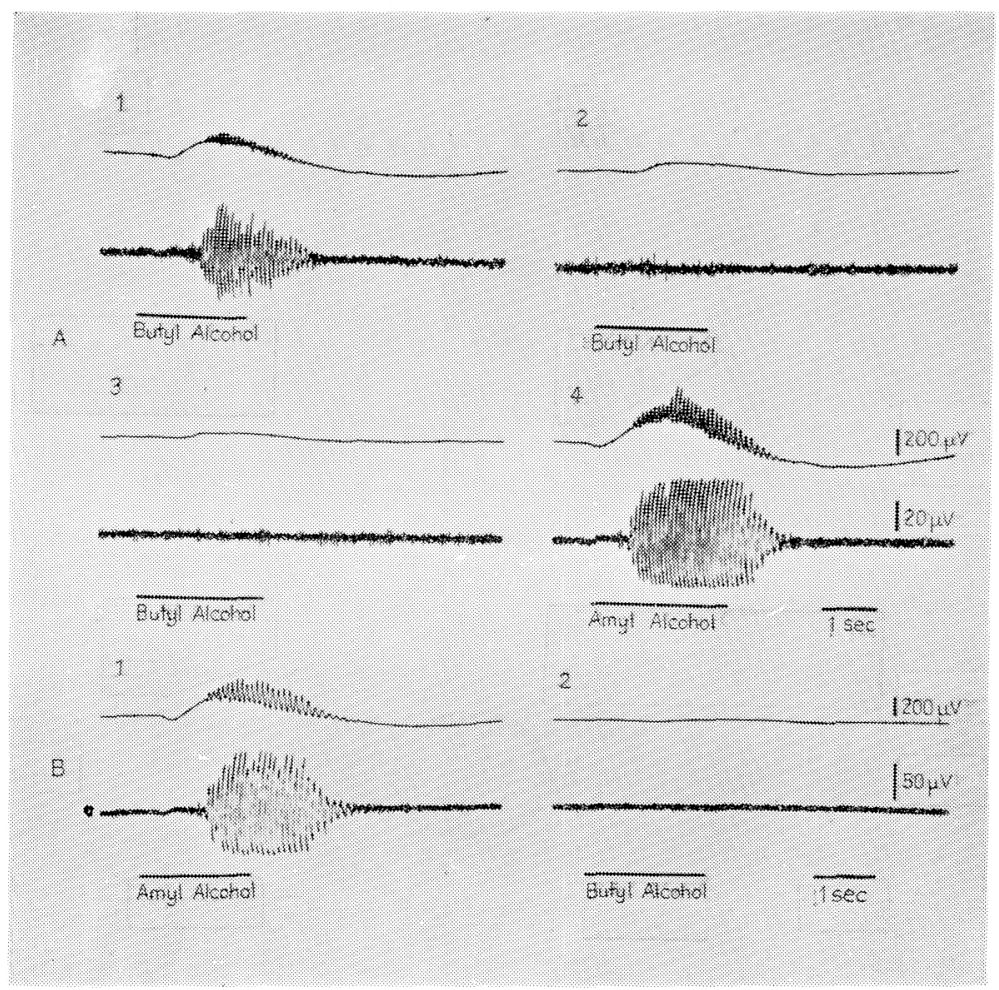

FIG. 6. The kinds of odours. A: The potential oscillation appeared in response to the application of butyl alcohol vapour in 1 . When the vapour was applied repetitively with an interval of 8 seconds, it disappeared as shown in 2 and 3 . The oscillation was reproduced with bigger magnitude by the application of amyl alcohol as shown in 4. B : Butyl alcohol vapour applied after amyl alcohol vapour did not reproduce the potential oscillation. A horizontal line under each record shows the time of olfactory stimulation. The top and bottom records were obtained by the same method as in FiG. 3. Detailed explanation in the text. 
studied. For instance, when the oscillation disappeared by repetitive application of butyl-alcohol, it was reproduced even with a bigger magnitude by the application of amyl-alcohol (FIG. $6 \mathrm{~A}$ ). Incidentally, a parallel relation was found between the appearance of the slow potential and the oscillation. However, when the order of application was reversed, the application of butyl-alcohol after amyl-alcohol was not effective in producing both the oscillation and the slow potential (FIG. 6 B). It was shown that amyl-alcohol produces larger slow potential than butyl-alcohol (OtToson, 1958). The above finding may occur because the odorants which produce larger slow potentials leave longer after-effects (Higashino, TAKAgI \& YAJima, unpublished). It was again found that the potential oscillation is produced only when the slow potential reaches a certain magnitude.

When we sniff a smell repetitively, we soon don't feel it at all. Nevertheless, we can smell other kind of odours. Since the odours of these two alcohols are different, the above finding is against our experience of selective adaptation.

VIII. Successive stimulation with different odours; When two different odours were applied successively, the second slow potential often summed on the first one and attained a larger magnitude. In such a case, the potential oscillation often appeared superimposed on the second slow potential (FIG. 7 A). This coincides well with the finding that the potential oscillation often appeared superimposed on the off-slow potential which summed on the on-slow potential (TAKAGI \& ShIBUYA, 1960 d). It is interesting that the oscillation appears on the slow potential of larger amplitude. Again, it was shown that the oscillation appears when the slow potential reaches a certain magnitude. How-

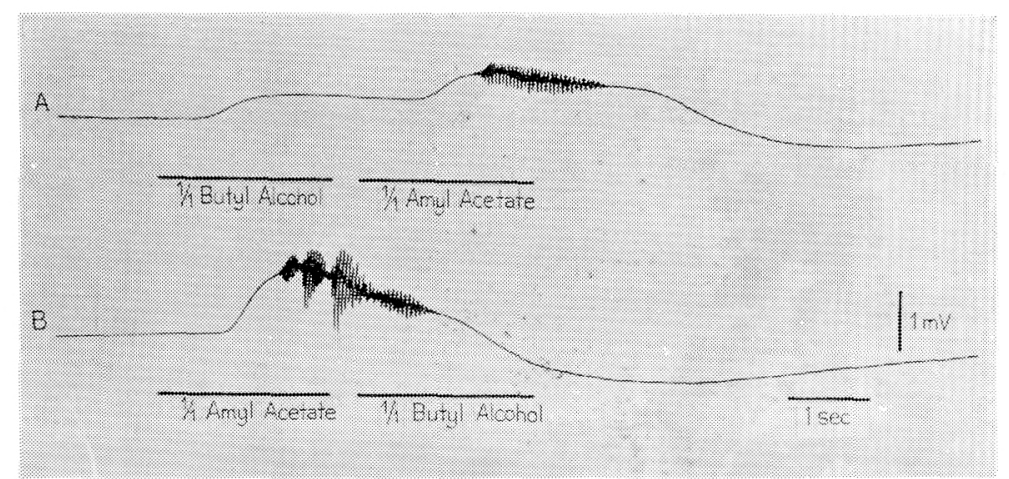

FIG. 7. Successive stimulation with different odours. A: Amyl acetate vapour applied after butyl alcohol vapour produced the second slow potential summed on the first one. A potential oscillation appeared superimposed on the second slow potential. B: Butyl alcohol applied after amyl acetate did not produce both the potential oscillation and the slow potential. Detailed explanation in the text. 


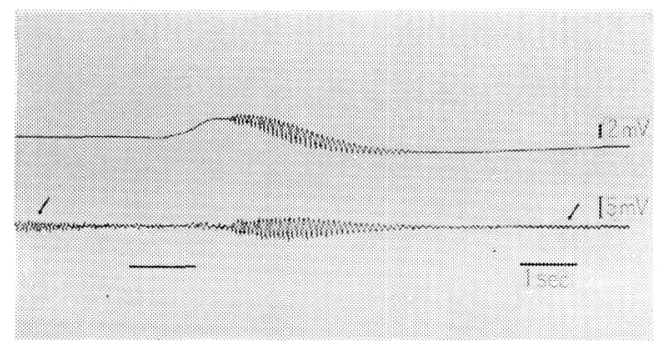

FIG. 8. Spontaneous potential oscillation. Arrows indicate the potential oscillation appearing spontaneously. The oscillation induced by olfactory stimulation is shown in the center. The horizontal line indicates the application of amyl acetate vapour.

ever, when the above two odours were applied inversely, the second odour did produce neither the potential oscillation nor the slow potential (FIG. 7B). A parallel relation was again found between the appearance of the potential oscillation and that of the slow potential. It was shown that the ability of an odorant to produce the oscillation can be compared among various kinds of odorants by this successive application method. The results will be stated in a following paper (Higashino, TAKAGI \& YAJIMA, unpublished).

IX. Spontaneous potential oscillation; The similar potential oscillations infrequently appeared spontaneously or after repeated olfactory stimulation. They continued as waxing and waning waves for considerably long time. They were recorded both with macro- and micro-electrodes (FIG. 8). Their magnitudes were always far smaller than those produced by odour stimulation and their frequencies were about 10 to $13 / \mathrm{sec}$.

$X$. Electrical stimulation; When an electrical pulse with $0.3 \mathrm{msec}$ duration was singly or repetitively ( 1 to $30 / \mathrm{sec}$ ) applied on the olfactory epithelium, a potential wave was evoked in the olfactory bulb, but neither the potential oscillation nor the slow potential could be produced in the olfactory epithelium. The same was the case, when the olfactory nerve was similarly stimulated. Besides, the potential oscillation produced by odour stimulation did not receive any appreciable influence from direct electrical stimulation or from the arrival of the antidromic impulses produced by olfactory nerve stimulation (FIG. 9).

Antidromic olfactory nerve impulses might be blocked in the transitory part between the olfactory nerve fibre and the olfactory cell, where the nerve fibre becomes suddenly thicker (ECCLES, 1953). If so, it is not strange that the potential oscillation and the slow potential were not affected by antidromic stimulation. Since the olfactory nerve can be stimulated electrically, it is concluded that both the potential oscillation and the slow potential are generated in the layer of specific olfactory receptors, but not in the olfactory nerve fibres. $X I$. Recordings in the partially covered eminentia; Before and after a part of the olfactory eminentia was covered with a thin vinyl membrane, the potential 
oscillations were recorded in the exposed part. When the posterior half of the eminentia was covered, the potential oscillations did not receive any appreciable influence. But when the anterior half was covered, the oscillations became far smaller than the controls. Similarly, the oscillations became far smaller, when the medial part was covered. Thus, the oscillation appeared more markedly in the anterio-medial part of the eminentia. This coincides well with the mode of generation of the slow potential (TAKAgI \& SHIBUYA, 1960 a).

Next, the olfactory eminentia was covered, leaving only a small area of $2 \mathrm{~mm}^{2}$. The potential oscillation recorded at the area before covering entirely disappeared (FIG. 10). This finding shows that the oscillation can not be produced by the activity of a small olfactory area, but only by the summation and interaction of many elementary oscillations in the broad area.

By means of a glass pipette with an inside diameter of $1.5 \mathrm{~mm}$, a small area was segregated from the remaining area of the eminentia (FIG. 11). The potential oscillation recorded at the same site became far smaller after segregation than before. It is interesting that the oscillations recorded in the neighbourhood of the glass pipette also decreased their amplitudes. Such a decrease

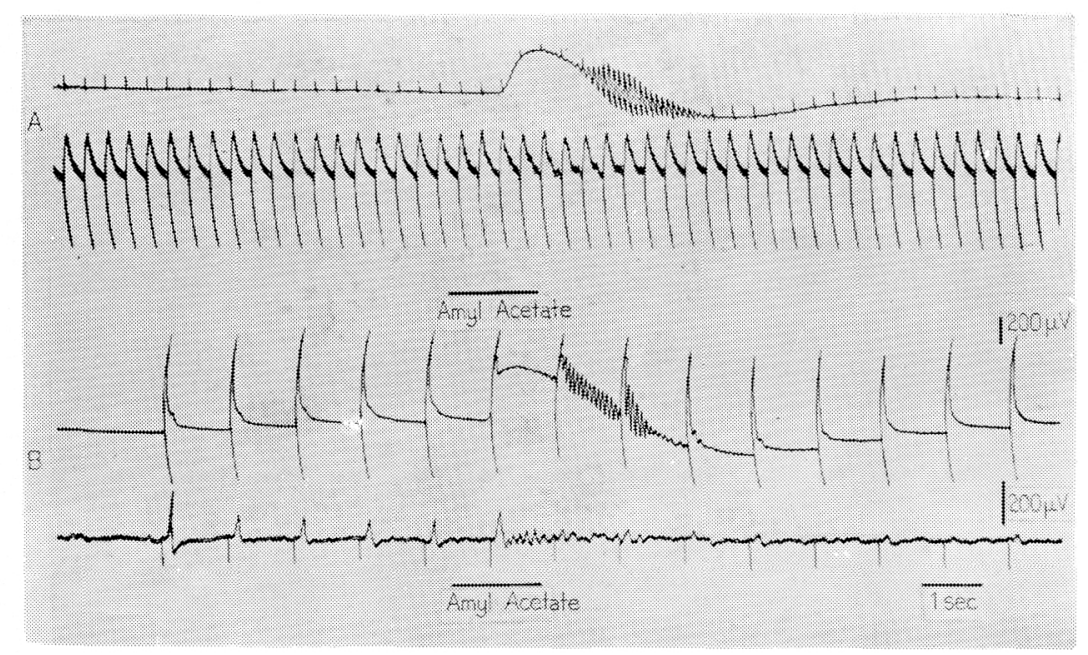

FIG. 9. The effect of electrical stimulation on the potential oscillation. A : Repetitive electrical stimulation was applied on the olfactory nerve. The top record shows the artifacts of electrical stimulation and the potential oscillation superimposed on the slow potential produced by amyl acetate vapour. The bottom record shows the evoked potential in the olfactory bulb. B: Repetitive electrical stimulation was applied on the olfactory epithelium. The top record shows the artifacts of electrical stimulation and the oscillation produced by amyl acetate vapour. The bottom record shows the evoked potential in the olfactory bulb. Thus, the potential oscillation produced by the application of an odour was not affected by electrical stimulation. 
in the amplitude is supposed to be due to the loss of the contribution from the segregated area. It was again shown that the oscillation is produced by the interaction and summation of many elementary oscillations.

$X I I$. Recording by micro-electrodes; By means of micro-electrodes, it was intended to find such an elementary potential oscillation. One or two microelectrodes were inserted as close as possible to a macro-electrode. The shapes and magnitudes of the oscillations recorded by the three electrodes were always similar, and there was not found any essential difference among them. Thus, it was not possible to find an elementary potential oscillation even with microelectrodes.

Instead, small potential discharges were often recorded with microelectrodes. They appeared coincidentally with the slow potential and often continued longer than stimulation. When the intensity of an odour was increased, the discharges were increased in number and often were replaced by potential oscillations (FIG. 12). It was found that the discharges disappear while the oscillation appears, but they reappear when the oscillation disappears. An elementary oscillation was presumed to occur in the olfactory epithelium $(I I, X I)$. It seems very probable that such an elementary potential oscillation

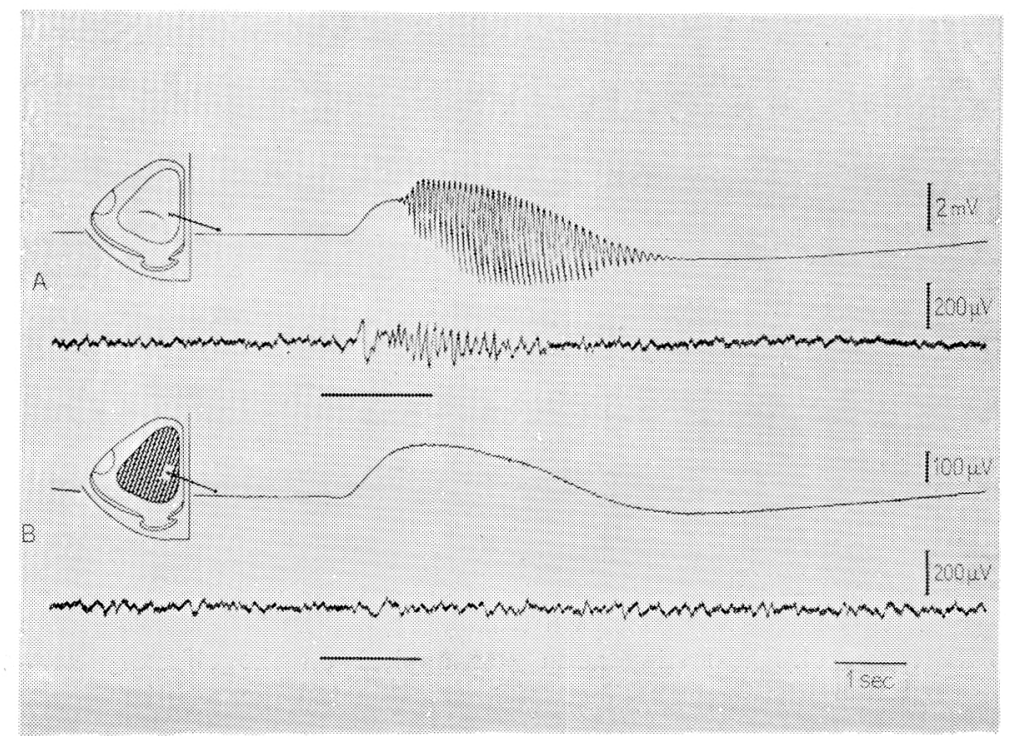

FIG. 10. Recordings in the partially exposed olfactory eminentia. When the olfactory eminentia was covered with a thin vinyl membrane, leaving only an area of $2 \mathrm{~mm}^{2}$, the oscillation recorded therein before disappeared together with the slow potential. They both can be made visible only with twenty times amplification in $\mathrm{B}$. The induced wave in $\mathrm{A}$ became invisible in B. The horizontal lines on the left below indicate the application of amyl acetate vapour. 


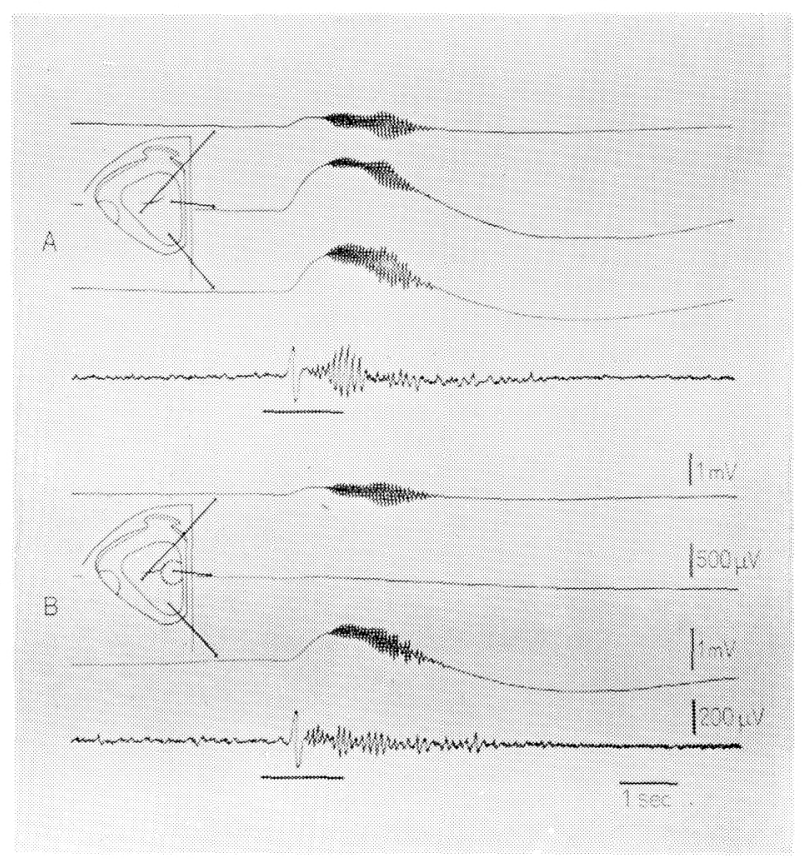

FIG. 11. Recording within an area segregated from olfactory stimulation. A: The potential oscillations recorded at three points on the eminentia. B : After a small area was segregated with a glass pipette from the rest of the olfactory eminentia, the oscillation recorded therein before decreased its amplitude considerably. The neighbouring oscillations also decreased their amplitudes slightly. The induced wave shown in the bottom record of A decreased in B apparently due to the decrease of the stimulated area.

is composed of these potential discharges in synchronization. The origin of the potential discharges has not been clarified because of the very small sizes of the cells and the fibres in the olfactory epithelium (ALLISON, 1953). It is only clear that they are different in origin from the action potentials of the olfactory cells, since the olfactory nerve discharges and the induced waves of the olfactory bulb appear and disappear much earlier than the above potential discharges, and there has not been found any relation between the two kinds of potentials.

\section{DISCUSSION}

The various properties of the potential oscillation in the olfactory epithelium were clarified in the present work. A resemblance may be found only in the retina (Fröhlich, 1914; KoKetsu, 1950; Svaetichin, 1952), but the latter is not so striking as the former, and may be different in origin.

The 'rhythmic waves' found by Оттоsоn (1956) in the olfactory epithelium 


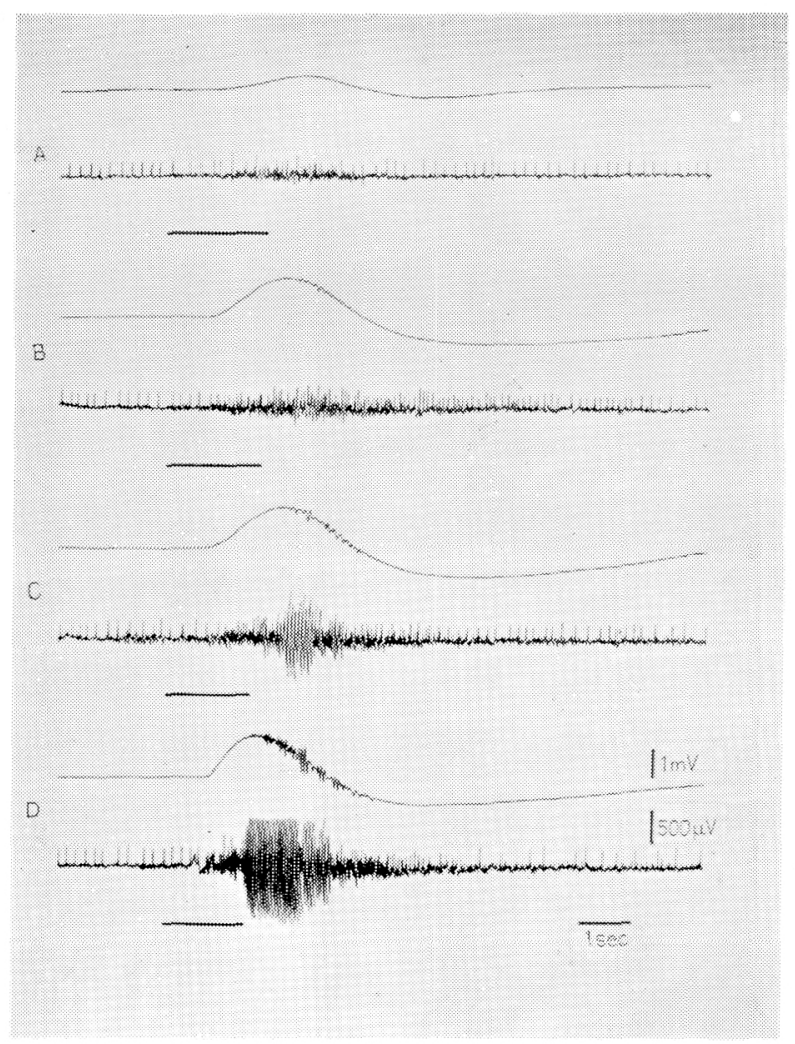

FIG. 12. The potential oscillation and the slow potential (Top) and the potential discharges recorded by micro-electrode (Bottom). The intensity of amyl acetate vapour was increased from $A$ to $D$. The slow potential increases its amplitude, and the potential oscillation begins to appear in $\mathbf{B}$. The potential discharges increase in number from $A$ to $D$. It seems that they are synchronized into a potential oscillation in C and D. A horizontal line on the left below indicates the application of the odour respectively.

of the frog, Rana temporaria, resemble the potential oscillations in the toad. Only the difference was that the frequency during a stimulation was constant in the former but decreased towards the end in the latter. In order to advance the comparison, the rhythmic waves were studied in the Japanese frog, Rana nigromaculata. They showed entirely the same features as the oscillation of the toad (TaKagi \& Shibuya, 1960 d). Consequently, it is concluded that the two potential oscillations in the toad and the frog are generated by a same mechanism in the epithelium.

ADRIAN $(1950,1954)$ found that the induced wave in the olfactory bulb of the rabbit is a kind of potential oscillation. Later, he (1955) found a similar oscillation in the olfactory epithelium of the rabbit. Considering that the potential pattern in the epithelium has all the features of that appearing 
in the bulb, he concluded that the two potentials are of identical origin. However, in the olfactory epithelium of the toad, we have not been able to find a potential oscillation comparable to the induced wave (TAKagi \& Shibuya, 1960 d). Consequently, our result in the amphibians does not support ADRIAN's conclusion. The similarity between the two potentials found in the rabbit may be fortuitous, and needs further experimentation in the future.

The role of the potential oscillation in olfaction was studied by applying various kinds of odours $(V I I)$. However, the mode of appearance of the oscillation did not coincide with our experience of selective olfactory adaptation. It is not known whether selective adaptation occurs in the toad. But if it is supposed to occur in the amphibian as in the human, the finding in VII does not show that the oscillation plays any active role in olfactory sensation.

The features of the potential oscillation are summarized as follows: 1) The oscillation is a local phenomenon in the olfactory epithelium and is not related in magnitude, frequency, shape and time of appearance to the afferent discharges in the olfactory nerves which convey olfactory messages (TAKAGI \& ShibuYA, 1960 d). 2) The potential oscillation begins to appear when the slow potential increases in amplitude beyond a certain magnitude $(I I I, I V, V I I, X I)$. Thus, it seems that there is a threshold magnitude of the slow potential in each part of the epithelium for the appearance of the oscillation. 3) The shapes, frequencies, magnitudes and times of appearance of the potential oscillations are different, depending on the recording site on the epithelium $(I I)$. 4) Small potential discharges recorded by micro-electrodes in the olfactory epithelium increased in number and it seems that they finally became synchronized into a potential oscillation when the intensity of an odorous vapour was gradually increased $(X I I)$. In the light of these findings, it was concluded that the potential oscillations of various shapes are produced by the interaction and summation of the potential discharges which occur with different magnitudes and frequencies at various areas.

Generator potentials have been found in various sensory receptors (GRANIT, 1947; Gray \& Sato, 1953; Katz, 1950; Eyzaguirre \& Kuffler, 1955). It was supposed that the slow potential in the olfactory epithelium is also a generator potential which produces repetitive olfactory nerve discharges. Since the olfactory cell is a sole neurone in the olfactory epithelium, this cell is believed to be the origin of the olfactory nerve discharge. It was stated before that the olfactory nerve discharge is not related to the potential oscillation in many points (TAKAGI \& SHIBUYA, 1960, c, d). Besides, small potential discharges which are believed to be the elements of the potential oscillation appeared indifferently to the olfactory nerve discharges. These findings strongly suggest that the potential oscillation originates in other elements than the olfactory cells. It is known that the olfactory pigment has a close relation with the acuity of olfaction (Allison, 1953; ONAGAwA, 1957). Since the 
pigment is found in the sustentacular cell, another cellular element in the olfactory epithelium, it is conceivable that the cell plays some kind of important role in olfaction and hence produces some kind of potential in response to olfactory stimulation. In the present stage of our research, it is not possible to find any other origin of the potential oscillation in the olfactory epithelium than in the sustentacular cells. The potential oscillation may possibly be produced in these cells by the electrotonic action of the slow potential.

\section{SUMMARY}

A potential oscillation appears superimposed on the slow potential in the olfactory epithelium of the toad, when an odorous vapour is applied. The properties and generative mechanism of the oscillation were studied.

1. The magnitudes and in many cases the frequencies of the oscillations increased with increasing stimulus intensity or increasing stimulus velocity $(I I I, I V)$. The frequency decreased linearly or exponentially towards the end during a stimulation (I).

2. The shapes, frequencies, durations and magnitudes of the oscillations are different not only in each toad but also at various sites on the olfactory epithelium of the same toad $(I I)$.

3. The durations of the oscillations were elongated with an exponential relation to the durations of olfactory stimulation $(V)$.

4. During repetitive olfactory stimulation, the magnitude, duration and frequency gradually decreased, and even the shape was changed. Eventually, the oscillation disappeared $(V I)$. The oscillation easily shows a fatigue-phenomenon.

5. The potential oscillations appeared differently depending on the kinds of odours $(V I I, V I I I)$.

6. The potential oscillation was neither produced nor affected by a single or repetitive electrical stimulation of the olfactory epithelium or nerve $(X)$.

7. By covering a part of the epithelium with a vinyl membrane, it was shown that the medial and anterior part of the eminentia was most important for the generation of the oscillation and that the oscillation was not produced by the activity of only a small olfactory area $(X I)$.

8. It was always found that the potential oscillation appears when the slow potential reaches a certain magnitude (III, IV, VII, XI).

9. Small potential discharges were found in the epithelium by micro-electrode. They increased in number and seemingly became synchronized into a potential oscillation with increasing stimulus intensity $(X I I)$.

10. It was concluded that the potential oscillations of various magnitudes and shapes are produced by the interaction and summation of the potential discharges recorded by micro-electrode in the olfactory epithelium. A generative mechanism of the potential oscillation was considered.

11. It was also concluded that the potential oscillation does not play any active role in olfactory sensation (VII). 
The authors are very much indebted to the Rockefeller Foundation for the contribution of some apparatus used in this experiment. Thanks are also due to Miss T. YAJIMA and Miss M. SAKAMOTo for the preparation of figures. This work was carried out with the aid of a grant for scientific research from the Ministry of Education.

\section{REFERENCES}

1) AdRIAN, E. D. (1950). The electrical activity of the mammalian olfactory bulb. Electroenceph. clin. Neurophysiol. 2: 377-388.

2) Adrian, E. D. (1954). Synchronized discharges from the organ of Jacobsen. J. Physiol. 126: $28-29$.

3) Adrian, E. D. (1955). Potential oscillations in the olfactory organ. J. Physiol. 128 : 21-22.

4) Allison, A. C. (1953). The morphology of the olfactory system in the vertebrates. Biol. Rev. 28: 195-244.

5) ECCLES, J. C. (1953). Neurophysiological Basis of Mind.. London: Oxford University Press.

6) Eyzaguirre, C. And Kuffler, S. W. (1955). Process of excitation in the dendrites and in the soma of single isolated sensory nerve cells of the lobster and crayfish. $J$. gen. Physiol. 39: 87-119.

7) FRöHLICH F. W. (1914). Beiträge zur allgemeinen Physiologie der Sinnesorgane. Z. Sinnesphysiol. 48: 28-164.

8) Granit, R. (1947). Sensory Mechanism of the Retina, p. 123. London: Oxford University Press.

9) Gray, J. A. B. AND Sato, M. (1953). Properties of the receptor potentials in pacinian corpuscles. J. Physiol. 122: 610-636.

10) Katz, B. (1950). Depolarization of sensory terminals and the inhibition of impulses in the muscle spindle. J. Physiol. 111: 261-282.

11) Koketsu, K. (1950). On the periodic changes of action potentials of retina of frog. J. Physiol. Soc. Japan. 12: 179-184.

12) Onagawa, K. (1957). Studies on the property of the olfactory mucous membrane for the sensibility of olfactory stimulation. J. Physiol. Soc. Japan. 19: 189-193.

13) Ottoson, D. (1956). Analysis of the electrical activity of the olfactory epithelium. Acta physiol. scand. 35: suppl. 122, 1-83.

14) Ottoson, D. (1958). Studies on the relationship between olfactory stimulating effectiveness and physico-chemical properties of odorous compounds. Acta physiol. scand. 43: $167-181$.

15) Svaetichin, G. (1952). The cone action potential. Acta physiol. scand. 29: suppl. 106, 565-600.

16) Takagi, S. F. And Shibuya, T. $(1960 a)$. The electrical activity of the olfactory epithelium studied with micro- and macro-electrodes. Jap. J. Physiol. 10: 385-395.

17) Takagi, S. F. And Shibuya, T. (1960b). The electrical activity of the lower olfactory nervous system of hte toad. In Electrical Activity of Single Cels. 1-10.

18) Takagi, S. F. ANd Shibuya, T. (1960c). Potential oscillations in the lower olfactory pathway of the toad. Nature, Lond.

19) Takagi, S. F. AND ShibuYa, T. $(1960 d)$. The potential oscillations observed in the olfactory epithelium, nerve and bulb of the toad and frog. Jap. J. Physiol. 10: 499-508.

20) Takagi, S. F., Shibuya, T., Higashino, S. and Arai, T. (1960). The stimulative and anaesthetic actions of ether on the olfactory epithelium of the frog and the toad. Jap. J. Physiol. 10: 571-584.

21) Tomita, T. (1956). The nature of action potentials in the lateral eye of the horseshoe $\mathrm{crab}$ as revealed by simultaneous intra- and extra-cellular recording. Jap. J. Physiol. 6: $327-340$. 